\title{
Present status of the KADoNiS database
}

\author{
Iris Dillmann*i \\ Forschungszentrum Karlsruhe, Institut für Kernphysik, Postfach 3640, D-76021 Karlsruhe and \\ Departement Physik und Astronomie, Universität Basel, Klingelbergstrasse 82, CH-4056 Basel \\ E-mail: iris.dillmann@ik.fzk.de
}

\section{Ralf Plag, Michael Heil, and Franz Käppeler}

Forschungszentrum Karlsruhe, Institut für Kernphysik, Postfach 3640, D-76021 Karlsruhe

\section{Thomas Rauscher}

Departement Physik und Astronomie, Universität Basel, Klingelbergstrasse 82, CH-4056 Basel

\begin{abstract}
The "Karlsruhe Astrophysical Database of Nucleosynthesis in Stars" (KADoNiS) project is an online database for experimental cross sections relevant to the $s$ process and $p$ process. It is available under http://nuclear-astrophysics.fzk.de/kadonis and consists of two parts. Part 1 is an updated sequel to the well-known Bao et al. compilations from 1987 and 2000, which is online since April 2005. An extension of this $s$-process database to $(n, p)$ and $(n, \alpha)$ cross sections at $k T=$ $30 \mathrm{keV}$, as in the first version of the Bao compilation, is planned. The second part of KADoNiS is a $p$-process library, which includes all available experimental data from $(p, \gamma),(p, n),(\alpha, \gamma)$, $(\alpha, n),(\alpha, \alpha),(n, \alpha)$ and $(\gamma, n)$ reactions in or close to the respective Gamow window. Despite the great number of reactions required for a $p$-process reaction network, experimental data is still scarce and up to now restricted to stable targets. Given here is a short overview about the present status of the KADoNiS database.
\end{abstract}

International Symposium on Nuclear Astrophysics - Nuclei in the Cosmos - IX

June 25-30 2006

CERN, Geneva, Switzerland

\footnotetext{
*Speaker.

${ }^{\dagger}$ This work was supported by the Swiss National Science Foundation Grants 2024-067428.01 and 2000-105328.
} 


\section{History of stellar neutron capture compilations}

The first collection of stellar neutron capture cross sections was published in 1971 by Allen and co-workers [1]. This paper reviewed the role of neutron capture reactions in the nucleosynthesis of heavy elements and presented also of a list of recommended (experimental or semi-empirical) Maxwellian averaged cross sections at $k T=30 \mathrm{keV}$ (MACS30) for nuclei between carbon and plutonium $(6 \leq \mathrm{Z} \leq 94)$. The idea of an experimental and theoretical stellar neutron cross section database was picked up again by Bao and Käppeler [2] for $s$-process studies. This compilation published in 1987 included cross sections for $(n, \gamma)$ reactions (between ${ }^{12} \mathrm{C}$ and ${ }^{209} \mathrm{Bi}$ ), some $(n, p)$ and $(n, \alpha)$ reactions $\left({ }^{33} \mathrm{Se}\right.$ to $\left.{ }^{59} \mathrm{Ni}\right)$, and also $(n, \gamma)$ and $(n, f)$ reactions for long-lived actinides. A (limited) updated compilation was published in 1992 by Beer, Voss and Winters [3].

In the update of 2000 [4] the Bao compilation from 1987 was extended to isotopes between ${ }^{1} \mathrm{H}$ and ${ }^{209} \mathrm{Bi}$, and listed - like the original Allen paper - also semi-empirical recommended values for nuclides without experimental cross section information. These estimated values are normalized cross sections derived with the Hauser-Feshbach code NON-SMOKER [5], which account for known systematic deficiencies in the nuclear input of the calculation. Additionally, the database provided stellar enhancement factors and energy-dependent MACS for energies between $k T=5$ $\mathrm{keV}$ and $100 \mathrm{keV}$.

\section{The updated big bang and $s$-process database}

The first update of the big bang and s-process database was finished in January 2006 [6]. In this stage, eight theoretical cross sections $\left({ }^{74} \mathrm{Se},{ }^{84} \mathrm{Sr},{ }^{128-130} \mathrm{Xe},{ }^{147} \mathrm{Pm},{ }^{151} \mathrm{Sm}\right.$, and $\left.{ }^{180} \mathrm{Ta}^{m}\right)$ were replaced with new experimental values. Further 20 cross sections were updated by the inclusion of new data. In order to make these updates reproducible, a full list of updated cross sections with references can be found on the KADoNiS homepage in the menu section "Logbook". Here will be also a history of previous KADoNiS versions.

The main future efforts in this part will be focussed on the re-evaluation of semi-empirical cross sections and the re-calculation of cross sections for isotopes, where a recent change in physical properties (e.g. $t_{1 / 2}, I_{\gamma \ldots}$ ) leads to changes in already measured cross sections. Another module will be the extension to $(n, p)$ and $(n, \alpha)$ cross sections at $k T=30 \mathrm{keV}$, as in the first version of the Bao compilation [2].

\section{The new $p$-process database}

The second part of KADoNiS is a collection of all experimental reaction rates relevant for $p$-process studies. The first stage is presented here and includes all available datasets of $(\alpha, \gamma)$ (Table 1) and $(p, \gamma)$ (Table 2) reactions within the respective Gamow window (see column $\mathrm{E}_{\text {Gamow }}$ ).

These tables are listed under http://nuclear-astrophysics.fzk.de/kadonis/pprocess, including hyperlinks to the respective EXFOR files. In the present stage (July 2006) we have included 39 $(p, \gamma)$ datasets of 32 isotopes between ${ }^{58} \mathrm{Ni}$ and ${ }^{119} \mathrm{Sn}$, and $9(\alpha, \gamma)$ reactions. For these isotopes we have created datasheets similar to those in the $s$-process database, which include tabulated cross sections data, from which the respective $S$ factor and reaction rate is calculated. As additional information, a graphical plot of the dataset(s) with the location of the Gamow window is given. A 


\begin{tabular}{cccccc}
\hline Isotope & Reaction & $\mathrm{E}_{\exp }[\mathrm{MeV}]$ & $\mathrm{E}_{\text {Gamow }}[\mathrm{MeV}]$ & Reference & EXFOR Entry \\
\hline${ }^{56} \mathrm{Fe}$ & $(\alpha, \gamma)$ & $3.90-6.50$ & $3.21-6.86$ & {$[7]$} & $\mathrm{A} 0308$ \\
${ }^{58} \mathrm{Ni}$ & $(\alpha, \gamma)$ & $4.90-6.10$ & $3.40-7.18$ & {$[8]$} & $\mathrm{C} 0703$ \\
${ }^{62} \mathrm{Ni}$ & $(\alpha, \gamma)$ & $5.10-8.60$ & $3.40-7.19$ & {$[9]$} & $\mathrm{C} 0669$ \\
${ }^{64} \mathrm{Ni}$ & $(\alpha, \gamma)$ & $4.40-7.10$ & $3.40-7.20$ & {$[9]$} & $\mathrm{C} 0669$ \\
${ }^{63} \mathrm{Cu}$ & $(\alpha, \gamma)$ & $5.90-8.70$ & $3.49-7.34$ & {$[10]$} & $\mathrm{C} 1050$ \\
${ }^{70} \mathrm{Ge}$ & $(\alpha, \gamma)$ & $5.05-7.80$ & $3.78-7.80$ & {$[11]$} & $\mathrm{O} 0897$ \\
${ }^{96} \mathrm{Ru}$ & $(\alpha, \gamma)$ & $7.03-10.56$ & $4.85-9.51$ & {$[12]$} & $\mathrm{A} 0451$ \\
${ }^{112} \mathrm{Sn}$ & $(\alpha, \gamma)$ & $8.30-9.97$ & $5.32-10.30$ & {$[13]$} & $\mathrm{C} 0904$ \\
${ }^{144} \mathrm{Sm}$ & $(\alpha, \gamma)$ & $10.50-13.40$ & $6.26-11.78$ & {$[14]$} & $\mathrm{A} 0416$ \\
\hline
\end{tabular}

Table 1: List of $(\alpha, \gamma)$ reactions within the Gamow window $\left(\mathrm{E}_{\text {Gamow }}\right)$, which are included in the $p$-process library.

graphical comparison with theoretical predictions from MOST [15] and NON-SMOKER [5] will also be included.

Up to the end of 2006 we plan to extend this database also to other available reactions, e.g. $(p, n),(p, \alpha),(\alpha, n),(\alpha, p),(n, \alpha)$ and $(n, p)$ reactions. A further step will be the inclusion of photodissociation rates, and the calculation of those rates from $(n, \gamma)$ reactions via detailed balance. Although KADoNiS is thought to be a "dynamic" database, which is updated regularly, a paper version will be published in 2007.

\section{References}

[1] B. Allen, J. Gibbons, and R. Macklin, Adv. Nucl. Phys., 4, (1971) 205.

[2] Z. Bao, and F. Käppeler, At. Data Nucl. Data Tables 36, (1987) 411.

[3] H. Beer, F. Voss, and R. Winters, Ap. J. Suppl. 80, (1992) 403.

[4] Z. Bao, H. Beer, F. Käppeler, F. Voss, K. Wisshak, and T. Rauscher, At. Data Nucl. Data Tables 76, (2000) 70.

[5] T. Rauscher and F.-K. Thielemann, At. Data Nucl. Data Tables 79, (2001) 47.

[6] I. Dillmann, M. Heil, F. Käppeler, R. Plag, T. Rauscher, and F.-K. Thielemann, AIP Conf. Proc. 819, (2006) 123.

[7] M.R. Anderson, S.R. Kennett, Z.E. Switkowski, D.G. Sargood, Nucl. Phys. A 316, (1979) 471.

[8] F.K. MacGowan, P.H. Stelson, W.G. Smith, Phys. Rev. B 133, (1964) 907.

[9] J.L. Zyskind, J.M. Davidson, M.T. Esat, M.H. Shapiro, R.H. Spear, Nucl. Phys. A 331, (1979) 180.

[10] M.S. Basunia, E.B. Norman, H.A. Shugart, A.R. Smith, M.J. Dolinski, and B.J. Quiter, Phys. Rev. C 71, (2005) 035801.

[11] Zs. Fülöp, A.Z. Kiss, E. Somorjai, C.E. Rolfs, H.P. Trautvetter, T. Rauscher, and H. Oberhummer, Z. Phys. A 355, (1996) 203.

[12] W. Rapp, M. Heil, D. Hentschel, F. Käppeler, R. Reifarth, H.J. Brede, H. Klein, and T. Rauscher, Phys. Rev. C 66, (2002) 015803. 
[13] N. Özkan, A.S.J. Murphy, R.N. Boyd, A.L. Cole, M. Famiano, R.T. Gray, M. Howard, L. Sahin, J.J. Zach, R. de Haan, J. Görres, M.C. Wiescher, M.S. Islam, and T. Rauscher, Nucl. Phys. A 710, (2002) 469.

[14] E. Somorjai, Zs. Fülöp, A.Z. Kiss, C.E. Rolfs, H.P. Trautvetter, U. Greife, M. Junker, S. Goriely, M. Arnould, M. Rayet, T. Rauscher, and H. Oberhummer, Astron. Astrophys. 333, (1998) 1112.

[15] S. Goriely, Hauser-Feshbach rates for neutron capture reactions (version 08/26/05), http://www-astro.ulb.ac.be/Html/hfr.html (2005).

[16] G.A. Krivonosov, B.A. Nemashkalo, O.I. Ekhichev, A.P. Klucharev, A.I. Popov, V.E. Storizhko, and V.K. Chirt, 24th Conference on Nuclear Spectroscopy and Nuclear Structure, Kharkov (USSR), 1974, p. 352 .

[17] C.W. Cheng and J.D. King, Can. Journ. of Physics 58, (1980) 1677.

[18] G.A. Krivonosov, B.A. Nemashkalo, O.I. Ekhichev, V.E. Storizhko, and V.K. Chirt, Izv. Rossiiskoi Akademii Nauk, Ser. Fiz. 41, Issue 10, (1977) 2196.

[19] C.W. Tingwell, L.W. Mitchell, M.E. Sevior, D.G. Sargood, Nucl. Phys. A 439, (1985) 371.

[20] M.E. Sevior, L.W. Mitchell, M.R. Anderson, C.W. Tingwell, D.G. Sargood, Austr. Journ. of Physics 36, (1983) 463.

[21] S. Qiang, Ph.D. thesis, University of Kentucky, Lexington/ USA, (1990).

[22] M.T. Esat, R.H. Spear, J.L. Zyskind, M.H. Shapiro, W.A. Fowler, and J.M. Davidson, Phys. Rev. C 23, (1981) 1822.

[23] Gy. Gyürky, Zs. Fülöp, E. Somorjai, M. Kokkoris, S. Galanopoulos, P. Demetriou, S. Harissopulos, T. Rauscher, and S. Goriely, Phys. Rev. C 68, (2003) 055803.

[24] Gy. Gyürky, E. Somorjai, Zs. Fülöp, S. Harissopulos, P. Demetriou, and T. Rauscher, Phys. Rev. C 64, (2001) 065803.

[25] S. Galanopoulos, P. Demetriou, M. Kokkoris, S. Harissopulos, R. Kunz, M. Fey, J.W. Hammer, Gy. Gyürky, Zs. Fülöp, E. Somorjai, and S. Goriely, Phys. Rev. C 67, (2003) 015801.

[26] P. Tsagari, M. Kokkoris, E. Skreti, A.G. Karydas, S. Harissopulos, T. Paradellis, and P. Demetriou, Phys. Rev. C 70, (2004) 015802.

[27] C.E. Laird, D. Flynn, R.L. Hershberger, and F. Gabard, Phys. Rev. C 35, (1987) 1265.

[28] F.R. Chloupek, A.S.J. Murphy, R.N. Boyd, A.L. Cole, J. Görres, R.T. Gray, G. Raimann, J.J. Zach, T. Rauscher, J.V. Schwarzenberg, P. Tischhauser, and M.C. Wiescher, Nucl. Phys. A 652, (1999) 391.

[29] S. Harissopulos, E. Skreti, P. Tsagari, G. Souliotis, P. Demetriou, T. Paradellis, J.W. Hammer, R. Kunz, C. Angulo, S. Goriely, and T. Rauscher, Phys. Rev. C 64, (2001) 055804.

[30] T. Sauter and F. Käppeler, Phys. Rev. C 55, (1997) 3127.

[31] J. Bork, H. Schatz, F. Käppeler, and T. Rauscher, Phys. Rev. C 58, (1998) 524. 


\begin{tabular}{|c|c|c|c|c|c|}
\hline Isotope & Reaction & $\mathrm{E}_{\exp }[\mathrm{MeV}]$ & $\mathrm{E}_{\text {Gamow }}[\mathrm{MeV}]$ & Reference & EXFOR Entry \\
\hline${ }^{58} \mathrm{Ni}$ & $(p, \gamma)$ & $1.32-2.74$ & $1.14-3.22$ & [16] & A0696 \\
\hline${ }^{58} \mathrm{Ni}$ & $(p, \gamma)$ & $1.00-4.91$ & $1.14-3.22$ & [17] & $\mathrm{C} 0886$ \\
\hline${ }^{58} \mathrm{Ni}$ & $(p, \gamma)$ & $0.51-3.09$ & $1.14-3.22$ & [18] & A0048 \\
\hline${ }^{58} \mathrm{Ni}$ & $(p, \gamma)$ & $1.14-4.09$ & $1.14-3.22$ & [19] & A0311 \\
\hline${ }^{60} \mathrm{Ni}$ & $(p, \gamma)$ & $0.61-2.94$ & $1.14-3.22$ & [18] & A0048 \\
\hline${ }^{61} \mathrm{Ni}$ & $(p, \gamma)$ & $1.11-2.94$ & $1.14-3.22$ & [18] & A0048 \\
\hline${ }^{64} \mathrm{Ni}$ & $(p, \gamma)$ & $1.11-2.94$ & $1.14-3.22$ & [20] & A0198 \\
\hline${ }^{63} \mathrm{Cu}$ & $(p, \gamma)$ & $1.11-4.69$ & $1.17-3.29$ & [20] & A0198 \\
\hline${ }^{63} \mathrm{Cu}$ & $(p, \gamma)$ & $1.99-4.52$ & $1.17-3.29$ & [21] & C0739 \\
\hline${ }^{65} \mathrm{Cu}$ & $(p, \gamma)$ & $1.03-3.22$ & $1.17-3.29$ & [20] & A0198 \\
\hline${ }^{65} \mathrm{Cu}$ & $(p, \gamma)$ & $1.99-4.52$ & $1.17-3.29$ & [21] & C0739 \\
\hline${ }^{64} \mathrm{Zn}$ & $(p, \gamma)$ & $1.47-2.73$ & $1.21-3.35$ & [18] & A0048 \\
\hline${ }^{67} \mathrm{Zn}$ & $(p, \gamma)$ & $1.47-2.92$ & $1.21-3.35$ & [18] & A 0048 \\
\hline${ }^{68} \mathrm{Zn}$ & $(p, \gamma)$ & $1.67-4.97$ & $1.21-3.35$ & [22] & $\mathrm{C} 0650$ \\
\hline${ }^{74} \mathrm{Se}$ & $(p, \gamma)$ & $1.60-3.00$ & $1.34-3.61$ & [18] & A0048 \\
\hline${ }^{74} \mathrm{Se}$ & $(p, \gamma)$ & $1.46-3.55$ & $1.34-3.61$ & [23] & O0849 \\
\hline${ }^{76} \mathrm{Se}$ & $(p, \gamma)$ & $1.46-3.55$ & $1.34-3.61$ & [23] & O0849 \\
\hline${ }^{77} \mathrm{Se}$ & $(p, \gamma)$ & $1.55-2.97$ & $1.34-3.61$ & [18] & A0048 \\
\hline${ }^{84} \mathrm{Sr}$ & $(p, \gamma)$ & $1.67-2.96$ & $1.47-3.85$ & [24] & A0426 \\
\hline${ }^{86} \mathrm{Sr}$ & $(p, \gamma)$ & $1.48-2.96$ & $1.47-3.85$ & [24] & A0426 \\
\hline${ }^{87} \mathrm{Sr}$ & $(p, \gamma)$ & $1.58-2.96$ & $1.47-3.85$ & [24] & A0426 \\
\hline${ }^{88} \mathrm{Sr}$ & $(p, \gamma)$ & $1.38-4.94$ & $1.47-3.85$ & [25] & O1054 \\
\hline${ }^{89} \mathrm{Y}$ & $(p, \gamma)$ & $1.76-4.83$ & $1.51-3.91$ & [26] & O1182 \\
\hline${ }^{90} \mathrm{Zr}$ & $(p, \gamma)$ & $1.97-5.70$ & $1.54-3.97$ & [27] & \\
\hline${ }^{96} \mathrm{Zr}$ & $(p, \gamma)$ & $3.50-6.00$ & $1.54-3.97$ & [28] & $\mathrm{C} 0556$ \\
\hline${ }^{93} \mathrm{Nb}$ & $(p, \gamma)$ & $1.42-4.80$ & $1.57-4.03$ & [29] & O0918 \\
\hline${ }^{92} \mathrm{Mo}$ & $(p, \gamma)$ & $1.48-3.00$ & $1.60-4.08$ & [30] & A0653 \\
\hline${ }^{94} \mathrm{Mo}$ & $(p, \gamma)$ & $1.48-2.49$ & $1.60-4.08$ & [30] & A0653 \\
\hline${ }^{95} \mathrm{Mo}$ & $(p, \gamma)$ & $1.70-3.00$ & $1.60-4.08$ & [30] & A0653 \\
\hline${ }^{98} \mathrm{Mo}$ & $(p, \gamma)$ & $1.48-3.00$ & $1.60-4.08$ & [30] & A0653 \\
\hline${ }^{96} \mathrm{Ru}$ & $(p, \gamma)$ & $1.65-3.37$ & $1.66-4.20$ & [31] & A0654 \\
\hline${ }^{98} \mathrm{Ru}$ & $(p, \gamma)$ & $1.65-3.37$ & $1.66-4.20$ & [31] & A0654 \\
\hline${ }^{99} \mathrm{Ru}$ & $(p, \gamma)$ & $1.46-3.37$ & $1.66-4.20$ & [31] & A0654 \\
\hline${ }^{100} \mathrm{Ru}$ & $(p, \gamma)$ & $1.46-3.37$ & $1.66-4.20$ & [31] & A0654 \\
\hline${ }^{104} \mathrm{Ru}$ & $(p, \gamma)$ & $1.65-3.37$ & $1.66-4.20$ & [31] & A0654 \\
\hline${ }^{102} \mathrm{Pd}$ & $(p, \gamma)$ & $2.53-4.17$ & $1.72-4.31$ & [13] & C0904 \\
\hline${ }^{112} \mathrm{Sn}$ & $(p, \gamma)$ & $3.00-8.50$ & $1.84-4.52$ & [28] & $\mathrm{C} 0556$ \\
\hline${ }^{116} \mathrm{Sn}$ & $(p, \gamma)$ & $2.63-4.18$ & $1.84-4.52$ & [13] & C0904 \\
\hline${ }^{119} \mathrm{Sn}$ & $(p, \gamma)$ & $2.80-6.00$ & $1.84-4.52$ & [28] & C0556 \\
\hline
\end{tabular}

Table 2: List of $(p, \gamma)$ reactions within the Gamow window $\left(\mathrm{E}_{\text {Gamow }}\right)$, which are included in the $p$-process library. 